\title{
Momentum distribution and condensate fraction of a Fermi gas in the BCS-BEC crossover
}

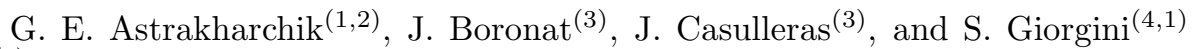 \\ (1) Dipartimento di Fisica, Università di Trento and BEC-INFM, I-38050 Povo, Italy \\ ${ }^{(2)}$ Institute of Spectroscopy, 142190 Troitsk, Moscow region, Russia \\ (3) Departament de Física i Enginyeria Nuclear, Campus Nord B4-B5, \\ Universitat Politècnica de Catalunya, E-08034 Barcelona, Spain \\ (4) JILA, University of Colorado, Boulder, CO 80309-0440, U.S.A.
}

(Dated: June 21, 2018)

\begin{abstract}
By using the diffusion Monte Carlo method we calculate the one- and two-body density matrix of an interacting Fermi gas at $T=0$ in the BCS-BEC crossover. Results for the momentum distribution of the atoms, as obtained from the Fourier transform of the one-body density matrix, are reported as a function of the interaction strength. Off-diagonal long-range order in the system is investigated through the asymptotic behavior of the two-body density matrix. The condensate fraction of fermionic pairs is calculated in the unitary limit and on both sides of the BCS-BEC crossover.
\end{abstract}

PACS numbers:

The physics of the crossover from Bardeen-CooperSchrieffer (BCS) superfluids to molecular Bose-Einstein condensates (BEC) in ultracold Fermi gases near a Feshbach resonance is a very exciting field that has recently attracted a lot of interest, both from the experimental [1, 2] and the theoretical side [3]. An important experimental achievement is the observation of a condensate of pairs of fermionic atoms on the side of the Feshbach resonance where no stable molecules would exist in vacuum [4, 5]. Although the interpretation of the experiment is not straightforward, as it involves an out-of-equilibrium projection technique of fermionic pairs onto bound molecules [6], it is believed that these results strongly support the existence of a superfluid order parameter in the strongly correlated regime on the BCS side of the resonance [5].

The occurrence of off-diagonal long-range order (ODLRO) in interacting systems of bosons and fermions was investigated by C.N. Yang in terms of the asymptotic behavior of the one- and two-body density matrix 7]. In the case of a two-component Fermi gas with $N_{\uparrow}$ spin-up and $N_{\downarrow}$ spin-down particles, the one-body density matrix (OBDM) for spin-up particles, defined as

$$
\rho_{1}\left(\mathbf{r}_{1}^{\prime}, \mathbf{r}_{1}\right)=\left\langle\psi_{\uparrow}^{\dagger}\left(\mathbf{r}_{1}^{\prime}\right) \psi_{\uparrow}\left(\mathbf{r}_{1}\right)\right\rangle
$$

does not possess any eigenvalue of order $N_{\uparrow}$. This behavior implies for homogeneous systems the asymptotic condition $\rho_{1}\left(\mathbf{r}_{1}^{\prime}, \mathbf{r}_{1}\right) \rightarrow 0$ as $\left|\mathbf{r}_{1}-\mathbf{r}_{1}^{\prime}\right| \rightarrow \infty$. In the above expression $\psi_{\uparrow}^{\dagger}(\mathbf{r})\left(\psi_{\uparrow}(\mathbf{r})\right)$ denote the creation (annihilation) operator of spin-up particles. The same result holds for spin-down particles. ODLRO may occur instead in the two-body density matrix (TBDM), that is defined as

$$
\rho_{2}\left(\mathbf{r}_{1}^{\prime}, \mathbf{r}_{2}^{\prime}, \mathbf{r}_{1}, \mathbf{r}_{2}\right)=\left\langle\psi_{\uparrow}^{\dagger}\left(\mathbf{r}_{1}^{\prime}\right) \psi_{\downarrow}^{\dagger}\left(\mathbf{r}_{2}^{\prime}\right) \psi_{\uparrow}\left(\mathbf{r}_{1}\right) \psi_{\downarrow}\left(\mathbf{r}_{2}\right)\right\rangle .
$$

For an unpolarized gas with $N_{\uparrow}=N_{\downarrow}=N / 2$, if $\rho_{2}$ has an eigenvalue of the order of the total number of particles $N$, the TBDM can be written as a spectral decomposition separating the largest eigenvalue,

$$
\rho_{2}\left(\mathbf{r}_{1}^{\prime}, \mathbf{r}_{2}^{\prime}, \mathbf{r}_{1}, \mathbf{r}_{2}\right)=\alpha N / 2 \varphi^{*}\left(\mathbf{r}_{1}^{\prime}, \mathbf{r}_{2}^{\prime}\right) \varphi\left(\mathbf{r}_{1}, \mathbf{r}_{2}\right)+\rho_{2}^{\prime},
$$

$\rho_{2}^{\prime}$ containing only eigenvalues of order one. The parameter $\alpha \leq 1$ in Eq. (3) is interpreted as the condensate fraction of pairs, in a similar way as the condensate fraction of single atoms is derived from the OBDM.

The spectral decomposition (3) yields for homogeneous systems the following asymptotic behavior of the TBDM

$$
\rho_{2}\left(\mathbf{r}_{1}^{\prime}, \mathbf{r}_{2}^{\prime}, \mathbf{r}_{1}, \mathbf{r}_{2}\right) \rightarrow \alpha N / 2 \varphi^{*}\left(\left|\mathbf{r}_{1}^{\prime}-\mathbf{r}_{2}^{\prime}\right|\right) \varphi\left(\left|\mathbf{r}_{1}-\mathbf{r}_{2}\right|\right)
$$

if $\left|\mathbf{r}_{1}-\mathbf{r}_{1}^{\prime}\right|,\left|\mathbf{r}_{2}-\mathbf{r}_{2}^{\prime}\right| \rightarrow \infty$. The complex function $\varphi$ is proportional to the order parameter $\left\langle\psi_{\uparrow}\left(\mathbf{r}_{1}\right) \psi_{\downarrow}\left(\mathbf{r}_{2}\right)\right\rangle=$ $\sqrt{\alpha N / 2} \varphi\left(\left|\mathbf{r}_{1}-\mathbf{r}_{2}\right|\right)$, whose appearance distinguishes the superfluid state of the Fermi gas. Equation (4) should be contrasted with the behavior of Bose systems with ODLRO, where $\rho_{1}$ has an eigenvalue of order $N$ [ $]$, and consequently the largest eigenvalue of $\rho_{2}$ is of the order of $N^{2}$.

In this Letter we present fixed-node diffusion Monte Carlo (FN-DMC) results of $\rho_{1}$ and $\rho_{2}$ for a homogeneous interacting Fermi gas at $T=0$ in the BCS-BEC crossover. From the Fourier transform of $\rho_{1}(r)$, we calculate the momentum distribution of the gas, $n_{\mathbf{k}}=$ $\int d^{3} \mathbf{r} \rho_{1}(r) e^{i \mathbf{k} \cdot \mathbf{r}}$, as a function of the interaction strength. From the asymptotic behavior of $\rho_{2}$, we extract the value of the condensate fraction of pairs $\alpha$. The calculated condensate fraction is compared with analytical expansions holding on the BEC and BCS side of the Feshbach resonance. The comparison with mean-field results [9] for $n_{\mathbf{k}}$ and $\alpha$ in the crossover region is also discussed.

We consider a homogeneous two-component unpolarized Fermi gas described by the Hamiltonian

$$
H=-\frac{\hbar^{2}}{2 m}\left(\sum_{i=1}^{N / 2} \nabla_{i}^{2}+\sum_{i^{\prime}=1}^{N / 2} \nabla_{i^{\prime}}^{2}\right)+\sum_{i, i^{\prime}} V\left(r_{i i^{\prime}}\right),
$$


where $m$ is the mass of the particles and $i, j, \ldots\left(i^{\prime}, j^{\prime}, \ldots\right)$ label spin-up (spin-down) particles. The strength of the interaction is assumed to be determined only by the parameter $1 / k_{F} a$, with $k_{F}=\left(3 \pi^{2} n\right)^{1 / 3}$ the Fermi wavevector fixed by the atomic density $n=N / V$, and $a$ the s-wave scattering length describing the low-energy collisions between the two fermionic species. The interatomic interactions in Eq. (5) are only between atoms with different spin and are modeled by a short-range potential that determines the value of $a$. In the present study, we use an attractive square-well potential, $V(r)=-V_{0}$ for $r<R_{0}$ and $V(r)=0$ otherwise, with $n R_{0}^{3}=10^{-6}$. We have verified that in the density range $n R_{0}^{3}=10^{-7}-10^{-5}$ the particular form of $V(r)$ is not relevant, and therefore the present results are in this sense universal. The different regimes: BEC $\left(a>0\right.$ and $\left.1 / k_{F} a \gg 1\right)$, BCS $(a<0$ and $\left.1 / k_{F}|a| \gg 1\right)$ and unitary limit $\left(1 / k_{F} a=0\right)$, are obtained by varying the potential depth $V_{0}$ as in [10]. Quantum Monte Carlo studies of the Hamiltonian (5) have already been carried out to investigate the $T=0$ equation of state 10, 11] and the pairing gap 11].

In a FN-DMC simulation, the wave function $f(\mathbf{R}, \tau)=$ $\psi_{T}(\mathbf{R}) \Psi(\mathbf{R}, \tau)\left(\mathbf{R}=\mathbf{r}_{1}, \ldots, \mathbf{r}_{N_{\uparrow}}, \mathbf{r}_{1^{\prime}}, \ldots, \mathbf{r}_{N_{\downarrow}}\right)$ is evolved in imaginary time $\tau=i t / \hbar$ according to the timedependent Schrödinger equation, with $\psi_{T}(\mathbf{R})$ acting as importance sampling function and as nodal constraint. The function $\Psi(\mathbf{R}) \equiv \Psi(\mathbf{R}, \tau \rightarrow \infty)$, which is the lowest energy state of the system having the nodes of $\psi_{T}(\mathbf{R})$, is obtained from the large-time evolution of $f(\mathbf{R}, \tau)$. The trial wave function we consider has the general form [11, 13] $\psi_{T}(\mathbf{R})=\Psi_{\mathrm{J}}(\mathbf{R}) \Psi_{\mathrm{BCS}}(\mathbf{R})$, where $\Psi_{\mathrm{J}}$ contains Jastrow correlations between all the particles,

$$
\Psi_{\mathrm{J}}(\mathbf{R})=\prod_{i<j} f_{\uparrow \uparrow}\left(r_{i j}\right) \prod_{i^{\prime}<j^{\prime}} f_{\downarrow \downarrow}\left(r_{i^{\prime} j^{\prime}}\right) \prod_{i, i^{\prime}} f_{\uparrow \downarrow}\left(r_{i i^{\prime}}\right),
$$

and the BCS-type wave function $\Psi_{\mathrm{BCS}}$ is the antisymmetrized product of the pair wave functions $\phi\left(\mathbf{r}_{i}-\mathbf{r}_{i^{\prime}}\right)$

$$
\Psi_{\mathrm{BCS}}(\mathbf{R})=\mathcal{A}\left(\phi\left(\mathbf{r}_{1}-\mathbf{r}_{1^{\prime}}\right) \phi\left(\mathbf{r}_{2}-\mathbf{r}_{2^{\prime}}\right) \ldots \phi\left(\mathbf{r}_{N_{\uparrow}}-\mathbf{r}_{N_{\downarrow}}\right)\right) .
$$

The pair orbital $\phi(\mathbf{r})$ is chosen as

$$
\phi(\mathbf{r})=\beta \sum_{k_{\alpha} \leq k_{\max }} e^{i \mathbf{k}_{\alpha} \cdot \mathbf{r}}+\phi_{s}(r),
$$

where the sum is performed over the plane wave states $\mathbf{k}_{\alpha}=2 \pi / L\left(\ell_{\alpha x} \hat{x}+\ell_{\alpha y} \hat{y}+\ell_{\alpha z} \hat{z}\right)$ up to the largest closed shell $k_{\max }=2 \pi / L\left(\ell_{\max }^{2}+\ell_{\operatorname{maxy}}^{2}+\ell_{\max z}^{2}\right)^{1 / 2}$ occupied by $N / 2$ particles. Here $L=V^{1 / 3}$ is the size of the cubic simulation box and $\ell$ are integer numbers. If $\phi_{s}(r)=0$ in Eq. (8), $\Psi_{\text {BCS }}$ in Eq. (7) coincides with the exact wave function of a free Fermi gas, i.e., the product of Slater determinants of spin-up and spin-down particles [14]. The spherically symmetric function $\phi_{s}(r)$ in Eq. (8) accounts for $s$-wave pairing. If $1 / k_{F} a \geq-0.2, \phi_{s}(\mathbf{r})$ corresponds to the solution of the two-body problem with the potential $V(r)$, as in Ref. [10]. For $1 / k_{F} a<-0.2$ we use instead $\phi_{s}(r)=\gamma_{1}\left[\exp \left(-\gamma_{2} r\right)+\exp \left(-\gamma_{2}(L-r)\right)\right]$. Here, $\gamma_{1}, \gamma_{2}$, and $\beta$ in Eq. (8) are variational parameters.
The Jastrow wave function $\Psi_{\mathrm{J}}$, Eq. (6), is determined as follows. For $1 / k_{F} a \geq-0.2$, we use $f_{\uparrow \downarrow}(r)=1$ and $f_{\uparrow \uparrow}(r)=f_{\downarrow \downarrow}(r)$ given by the two-body solution of a fictitious repulsive step potential with range $\tilde{R}$ and scattering length $\tilde{a}$. The boundary conditions $f(r=L / 2)=1$ and $f^{\prime}(r=L / 2)=0$ determine the wave function in terms of the variational parameters $\tilde{R}$ and $\tilde{a}$. For $1 / k_{F} a<-0.2$, we use instead $f_{\uparrow \uparrow}(r)=f_{\downarrow \downarrow}(r)=1$ and the model used in Ref. 10] for the crossed correlation factor, $f_{\uparrow \downarrow}(r)$. It is worth noticing that the function $\psi_{T}(\mathbf{R})$ defined above reproduces as a special case the trial wave function used in the preceding study [10], but contains more variational parameters. The parameters of the Jastrow function $\Psi_{\mathrm{J}}$, Eq. (6), are optimized by minimizing the variational expectation value $\left\langle\psi_{T}|H| \psi_{T}\right\rangle /\left\langle\psi_{T} \mid \psi_{T}\right\rangle$. The parameters of the BCS function $\Psi_{\mathrm{BCS}}$, Eq. (7), affect the nodal surface of the trial wave function and they are optimized by minimizing the FN-DMC estimate of the energy. For the values of $1 / k_{F} a$ used in the present study, the calculated FN-DMC energies are in agreement with the results obtained in 10], although the optimized variational energy has significantly improved.

A direct estimate of any operator $O$ in DMC is known as mixed estimate, $\langle O\rangle_{\mathrm{m}}=\left\langle\psi_{T}|O| \Psi\right\rangle /\left\langle\psi_{T} \mid \Psi\right\rangle$, and is exact only for the Hamiltonian and operators commuting with it. If $O$ is a local operator, one can circumvent this problem by introducing pure estimators. That is not the case for $\rho_{1}$ and $\rho_{2}$ which are the objectives of the present work. In order to reduce, and even eliminate in practice, a possible bias in the calculation we have used the extrapolated estimator $\langle\Psi|O| \Psi\rangle /\langle\Psi \mid \Psi\rangle \simeq 2\langle O\rangle_{\mathrm{m}}-\langle O\rangle_{\mathrm{v}}$, with $\langle O\rangle_{\mathrm{v}}=\left\langle\psi_{T}|O| \psi_{T}\right\rangle /\left\langle\psi_{T} \mid \psi_{T}\right\rangle$. Any residual bias in the extrapolated estimator is reduced if the trial function $\psi_{T}(\mathbf{R})$ has a large overlap with $\Psi(\mathbf{R})$. Consequently, compared to the calculation of the eigenenergies, the optimization of $\psi_{T}$ is a more important issue in the calculation of observables like the OBDM and TBDM [13].

We consider a system with $N=66$ particles and periodic boundary conditions. In Fig. 1 we show results of the OBDM $\rho_{1}(r)$, Eq. (11), for different values of the interaction strength $1 / k_{F} a$. In the deep molecular regime, $1 / k_{F} a \gg 1$, the OBDM is determined by the molecular wave function $\phi_{\mathrm{bs}}(r): \rho_{1}(r) \simeq n / 2 \int d^{3} \mathbf{r}^{\prime} \phi_{\mathrm{bs}}^{*}(\mid \mathbf{r}+$ $\left.\mathbf{r}^{\prime} \mid\right) \phi_{\mathrm{bs}}\left(r^{\prime}\right)$. For a zero-range potential the molecular wave function is given by $\phi_{\mathrm{bs}}(r) \propto e^{-r / a} / r$ and one finds $\rho_{1}(r) \simeq n e^{-r / a} / 2$. This behavior is shown in Fig. 1 for $1 / k_{F} a=4$. If one moves closer to the resonance, the OBDM decays slowly and oscillations start to appear. Finally, on the BCS side of the resonance, the OBDM becomes more and more similar to the ideal gas result $\rho_{1}(r)=3 n\left[\sin \left(k_{F} r\right) /\left(k_{F} r\right)-\cos \left(k_{F} r\right)\right] /\left[2\left(k_{F} r\right)^{2}\right]$. The momentum distribution $n_{\mathbf{k}}$, obtained from the Fourier transform of $\rho_{1}(r)$, is shown in Fig. 2 In the inset of Fig. 2] we compare $n_{\mathbf{k}}$, calculated using FN-DMC for $1 / k_{F} a=4$, with the momentum distribution of the atoms in the molecular state $n_{\mathbf{k}}=4\left(k_{F} a\right)^{3} /\left[3 \pi\left(1+k^{2} a^{2}\right)^{2}\right]$ [15]. To reduce finite-size effects in the calculation of the Fourier transform for $1 / k_{F} a=0$ and -1 , we have used 


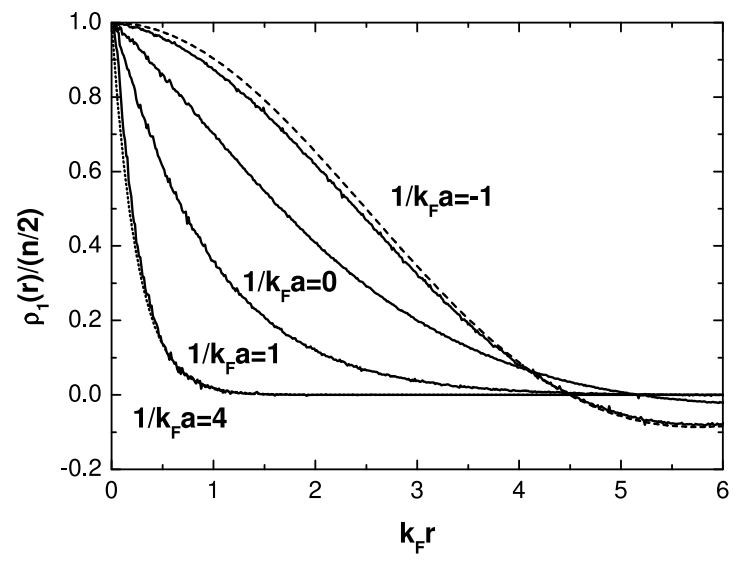

FIG. 1: OBDM for different values of the interaction strentgth $1 / k_{F} a$ (solid lines). The dotted line corresponds to $e^{-r / a}$ for $1 / k_{F} a=4$ and the dashed line is the OBDM of a non interacting gas.

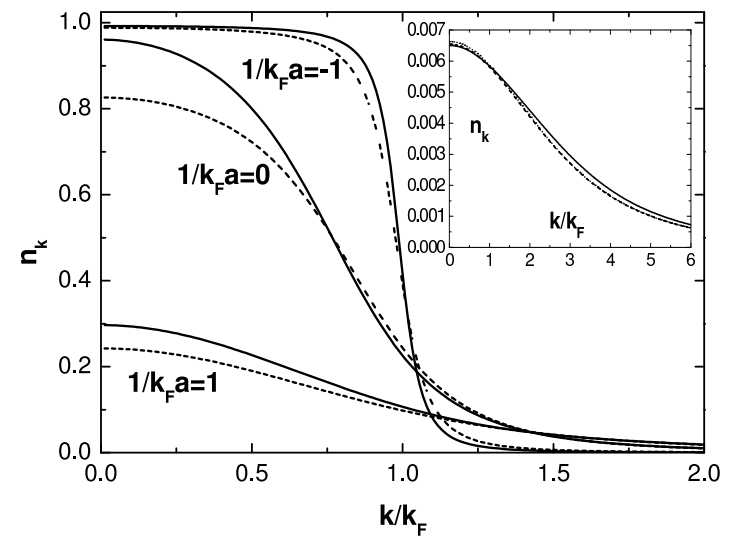

FIG. 2: Momentum distribution $n_{\mathbf{k}}$ for different values of $1 / k_{F} a$ (solid lines). The dashed lines correspond to $n_{\mathbf{k}}$ calculated using the BCS mean-field approach [15]. Inset: $n_{\mathbf{k}}$ for $1 / k_{F} a=4$. The dotted line corresponds to the momentum distribution of the molecular state (see text). It almost coincides with the mean-field result (dashed line).

the following model for the $k$-dependence of $n_{\mathbf{k}}$

$$
n_{\mathbf{k}}=A\left(1-\frac{\left(k / k_{F}\right)^{2}-\mu}{\sqrt{\left[\left(k / k_{F}\right)^{2}-\mu\right]^{2}+\Delta^{2}}}\right) .
$$

The values of $\mu, \Delta$ and $A$ are free parameters determined by the best fit of the inverse Fourier transform of Eq. (9) to the calculated $\rho_{1}(r)$, with the constraint $1 / V \sum_{k} n_{\mathbf{k}}=n / 2$. For $A=1 / 2$, the above expression reproduces the standard $n_{\mathbf{k}}$ of BCS theory with $\mu$ and $\Delta$, respectively, chemical potential and gap in units of the Fermi energy $\epsilon_{F}=\hbar^{2} k_{F}^{2} / 2 m$. For $1 / k_{F} a=0$ and -1 the Fourier transform of the BCS model, Eq. (9), reproduces quite well the calculated $\rho_{1}(r)$ with a $\chi^{2} / \nu$ of

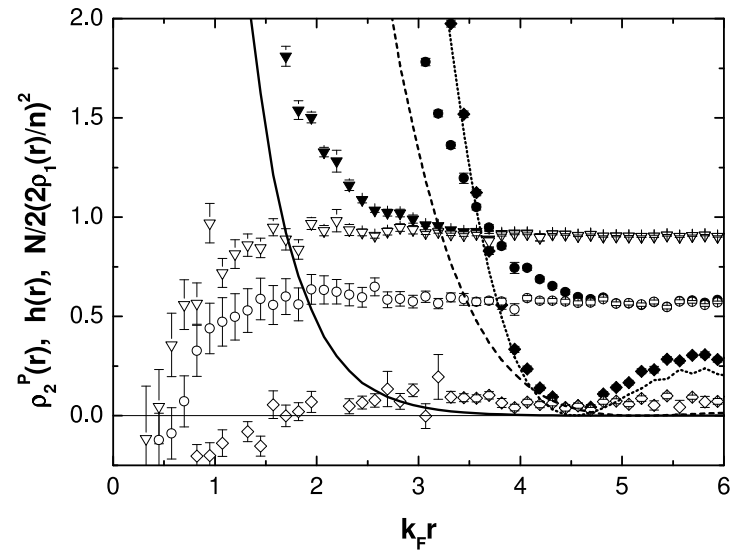

FIG. 3: Projected TBDM, $\rho_{2}^{P}(r)$, (solid symbols), $N / 2\left(2 \rho_{1}(r) / n\right)^{2}$ (lines) and $h(r)=\rho_{2}^{P}(r)-N / 2\left(2 \rho_{1}(r) / n\right)^{2}$ (open symbols) for different values of $1 / k_{F} a: 1 / k_{F} a=1$ (solid line and triangles), $1 / k_{F} a=0$ (dashed line and circles) and $1 / k_{F} a=-1$ (dotted line and diamonds).

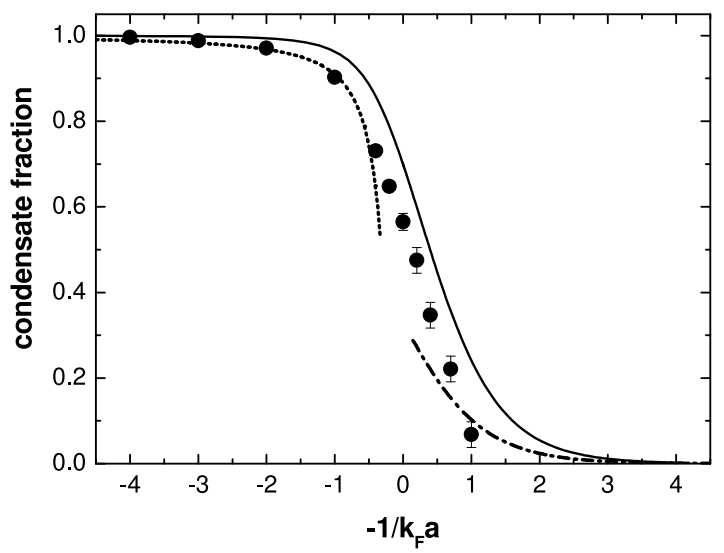

FIG. 4: Condensate fraction of pairs $\alpha$ as a function of the interaction strength: FN-DMC results (symbols), Bogoliubov quantum depletion of a Bose gas with $a_{m}=0.6 a$ (dashed line), BCS theory using Eq. (11) (dot-dashed line) and selfconsistent mean-field theory (solid line).

the order of one. In Fig. 2 we also show the results of $n_{\mathbf{k}}$ obtained using the BCS mean-field theory [9], where the values of chemical potential and gap are calculated selfconsistently through the gap and number equations [15]. As evident from Fig. 2] the mean-field theory sistematically overestimates the broadening of the momentum distribution, apart from the deep BEC regime where both mean-field and FN-DMC results coincide with the momentum distribution of the molecular state.

The condensate fraction of pairs has been obtained from the the projected TBDM, defined as [16, 17]

$$
\rho_{2}^{P}(r)=\frac{2}{N} \int d^{3} \mathbf{r}_{1} d^{3} \mathbf{r}_{2} \rho_{2}\left(\mathbf{r}_{1}+\mathbf{r}, \mathbf{r}_{2}+\mathbf{r}, \mathbf{r}_{1}, \mathbf{r}_{2}\right) .
$$


Assuming the eigenvector $\varphi(r)$ of Eq. (4) normalized to $1 / V$, the large $r$ behavior of $\rho_{2}^{P}(r)$ tends to the condensate fraction $\alpha$ : $\lim _{r \rightarrow \infty} \rho_{2}^{P}(r)=\alpha$. In terms of the order parameter, $F\left(\left|\mathbf{r}_{1}-\mathbf{r}_{2}\right|\right)=\left\langle\psi_{\uparrow}\left(\mathbf{r}_{1}\right) \psi_{\downarrow}\left(\mathbf{r}_{2}\right)\right\rangle$, one has instead $\lim _{r \rightarrow \infty} \rho_{2}^{P}(r)=2 / n \int d^{3} \mathbf{r}^{\prime}\left|F\left(r^{\prime}\right)\right|^{2}$. In Fig. 3 we show the results of $\rho_{2}^{P}(r)$. Finite size effects can be substantially reduced if one considers the following decomposition: $\lim _{r \rightarrow \infty} \rho_{2}\left(\mathbf{r}_{1}+\mathbf{r}, \mathbf{r}_{2}+\mathbf{r}, \mathbf{r}_{1}, \mathbf{r}_{2}\right)=\left|F\left(\left|\mathbf{r}_{1}-\mathbf{r}_{2}\right|\right)\right|^{2}+$ $\rho_{1}^{2}(r)$, accounting for the large $r$ behavior of $\rho_{2}$ when $\rho_{1}(r)$ is small but not zero. From this result one finds for the asymptotic behavior of the projected TBDM: $\lim _{r \rightarrow \infty} \rho_{2}^{P}(r)=\alpha+N / 2\left(2 \rho_{1}(r) / n\right)^{2}$. In Fig. B3 we show results for the quantity $h(r)=\rho_{2}^{P}(r)-N / 2\left(2 \rho_{1}(r) / n\right)^{2}$. For values of $1 / k_{F} a$ on the BEC side of the Feshbach resonance and up to the unitary limit $1 / k_{F} a=0$, the asymptotic values of $\rho_{2}(r)$ and $h(r)$ coincide. On the BCS side, instead, finite-size effects become visible in the large $r$ behavior of $\rho_{2}^{P}(r)$, but are strongly suppressed for $h(r)$. The results for the condensate fraction $\alpha$, as obtained from the asymptotic behavior of $h(r)$, are shown in Fig. 4 In the BEC regime, the results reproduce the Bogoliubov quantum depletion of a gas of composite bosons $\alpha=1-8 \sqrt{n_{m} a_{m}^{3}} / 3 \sqrt{\pi}$, where $n_{m}=n / 2$ is the density of molecules and $a_{m}=0.6 a$ is the dimer-dimer scattering length [10, 18]. In the opposite BCS regime, the condensate fraction $\alpha$ can be calculated from the result of the BCS order parameter holding for $r \gg a[19]$

$$
F_{\mathrm{BCS}}(r)=\frac{\Delta k_{F}^{3}}{\epsilon_{F}} \frac{\sin \left(k_{F} r\right)}{4 \pi^{2} k_{F} r} K_{0}\left(r / \xi_{0}\right)
$$

where $\xi_{0}=\hbar^{2} k_{F} / m \Delta$ is the coherence length and $K_{0}(x)$ is the modified Bessel function. If we include the Gorkov-Melik Barkhudarov correction for the pairing gap [20] $\Delta=(2 / e)^{7 / 3} \epsilon_{F} e^{-\pi / 2 k_{F}|a|}$, we obtain for $\alpha=2 / n \int d^{3} \mathbf{r} F_{\mathrm{BCS}}^{2}(r)$ the dot-dashed line shown in Fig. 4 For $1 / k_{F} a \leq-1$ the coherence length $\xi_{0}$ becomes increasingly larger and finite-size effects start to be relevant in the FN-DMC estimate of $\alpha$. For example at $1 / k_{F} a=-1$, the value of $\alpha$, as obtained from $F_{B C S}(r)$ [Eq. [11] ], reduces from 0.10 to 0.08 by cutting off the spacial integral at the simulation box size $r=L / 2$. The condensate fraction $\alpha$ can also be estimated in the crossover region using the self-consistent mean-field approach [9] and the result for the order parameter: $F(r)=1 / V \sum_{\mathbf{k}} u_{k} v_{k} e^{i \mathbf{k} \cdot \mathbf{r}}$, where $u_{k}$ and $v_{k}$ are the usual quasiparticle amplitudes of BCS theory. The result is shown in Fig. 4 with a solid line (see also Ref. 17]). The mean-field result reproduces the qualitative behavior of the condensate fraction across the resonance. However, it does not reproduce the quantum depletion in the BEC regime, nor the Gorkov-Melik Barkhudarov correction to the gap in the BCS regime.

In conclusion, we have investigated using quantum Monte Carlo techniques the one- and two-body density matrix of a homogeneous Fermi gas in the BCS-BEC crossover. Results for the momentum distribution are obtained as a function of the interaction strength. The condensate fraction of pairs is calculated from the asymptotic behavior of the two-body density matrix. The results obtained are in good agreement with the quantum depletion of a weakly-interacting Bose gas in the BEC regime and with the normalization of the BCS order parameter in the BCS regime.

Acknowledgements:GEA and SG acknowledge support by the Ministero dell'Istruzione, dell'Università e della Ricerca (MIUR). JB and JC acknowledge support from DGI (Spain) Grant No. BFM2002-00466 and Generalitat de Catalunya Grant No. 2001SGR-00222.
[1] M. Greiner, C.A. Regal and D.S. Jin, Nature (London) 426, 537 (2003); S. Jochim et al., Science 302, 2101 (2003); M.W. Zwierlein et al., Phys. Rev. Lett. 91250401 (2003).

[2] M. Bartenstein et al., Phys. Rev. Lett. 92, 120401 (2004); T.Bourdel et al., Phys. Rev. Lett. 93, 050401 (2004).

[3] See e.g. the recent review: Q. Chen, J. Stajic, S. Tan and K. Levin, cond-mat/0404274, and references therein.

[4] C.A. Regal, M. Greiner and D.S. Jin, Phys. Rev. Lett. 92, 040403 (2004); M.W. Zwierlein et al., ibid. 92, 120403 (2004).

[5] M.W. Zwierlein et al., Phys. Rev. Lett. 94, 180401 (2005).

[6] R.B. Diener and T.-L. Ho, cond-mat/0404517 A. Perali, P. Pieri and G.C. Strinati, cond-mat/0501631 E. Altman and A. Vishwanath, cond-mat/0501683

[7] C.N. Yang, Rev. Mod. Phys. 34, 694 (1962).

[8] O. Penrose and L. Onsager, Phys. Rev. 104, 576 (1956).

[9] A.J. Leggett, in Modern Trends in the Theory of Condensed Matter, edited by A. Pekalski and R. Przystawa (Springer-Verlag, Berlin, 1980); P. Nozières and S.
Schmitt-Rink, J. Low Temp. Phys. 59, 195 (1985); J.R. Engelbrecht, M. Randeria and C.A.R. Sá de Melo, Phys. Rev. B 55, 15153 (1997).

[10] G.E. Astrakharchik, J. Boronat, J. Casulleras and S. Giorgini, Phys. Rev. Lett. 93, 200404 (2004).

[11] J. Carlson, S.-Y. Chang, V.R. Pandharipande and K.E. Schmidt, Phys. Rev. Lett. 91, 050401 (2003); S.Y. Chang, V.R. Pandharipande, J. Carlson and K.E. Schmidt, Phys. Rev. A 70, 043602 (2004).

[12] D.M. Ceperley and M.H. Kalos, in Monte Carlo Methods in Statistical Physics, edited by K. Binder (Springer, Berlin, 1979).

[13] S.Y. Chang and V.R. Pandharipande, physics/0409060

[14] J.P. Bouchaud, A. Georges and C. Lhuillier, J. Phys. (Paris) 49, 553 (1988); J.P. Bouchaud and C. Lhuillier, Z. Phys. B 75, 283 (1989).

[15] L. Viverit, S. Giorgini, L.P. Pitaevskii and S. Stringari, Phys. Rev. A 69, 013607 (2004).

[16] S. De Palo, F. Rapisarda and G. Senatore, Phys. Rev. Lett. 88, 206401 (2002).

[17] G. Ortiz and J. Dukelsky, cond-mat/0503664 L. Salas- 
nich, N. Manini and A. Parola, cond-mat/0506074

[18] D.S. Petrov, C. Salomon and G.V. Shlyapnikov, Phys. Rev. Lett. 93, 090404 (2004).

[19] J. Bardeen, L.N. Cooper and J.R. Schrieffer, Phys. Rev.
108, 1175 (1957)

[20] L.P. Gorkov and T.K. Melik-Barkhudarov, Sov. Phys. JETP 13, 1018 (1961). 